\title{
Novo modelo de parâmetros concentrados aplicado à hidratação de grãos
}

New model of lumped parameters applied to grain hydration

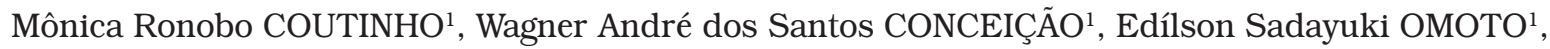 \\ Cid Marcos Gonçalves ANDRADE ${ }^{1}$, Luiz Mario de Matos JORGE ${ }^{1 *}$
}

\section{Resumo}

Este trabalho apresenta um novo modelo fenomenológico de parâmetros concentrados para a hidratação de grãos de soja, obtido a partir de um balanço de massa em regime transiente nos grãos, admitindo duas hipóteses básicas: $1^{\circ}$ ) variação do volume do grão diretamente proporcional à variação da sua massa ao longo da hidratação; e $2^{\circ}$ ) variação exponencial do coeficiente de transferência de massa aparente em função da concentração de água na soja. Este modelo possui apenas dois parâmetros, os quais foram estimados a partir do ajuste deste às medidas da umidade do grão ao longo do tempo, avaliadas em várias temperaturas. Os resultados revelam que o modelo proposto é adequado, enquanto o coeficiente de transferência de massa aparente depende da temperatura e apresenta variações significativas ao longo da hidratação.

Palavras-chave: soja; modelagem; hidratação.

\begin{abstract}
A new first principle model with lumped parameters was developed for the hydration of soya beans. A transient mass balance was applied to the soya beans taking into consideration two basic hypotheses: $1^{\circ}$ ) variation of the volume directly proportional to the variation of the mass of the grain during the process, $2^{\circ}$ ) Exponential variation of the apparent coefficient of mass transfer in function of water concentration in the soy. This model has got just two parameters, which were evaluated from adjustment of model to the measures of grain moisture along the time, at several temperatures. The results indicate that the proposed model was able to represent the hydration process, while the mass transfer coefficient depends on the temperature and it presents significant variations along the hydration.

Keywords: soy; modelling; hydration.
\end{abstract}

\section{Introdução}

Modelos para representar a hidratação de grãos têm sido desenvolvidos com o intuito de se prever o tempo necessário para se obter o grau de umidade desejado. O interesse no estudo deste processo se deve ao fato da hidratação de grãos de soja reduzir o tempo de cozimento, diminuir as perdas e melhorar a qualidade dos produtos obtidos a partir deste grão ${ }^{11}$. Além disso, a umidificação também está presente em estudos de secagem de cereais em escala de bancada ${ }^{3,1}$ e da capacidade germinativa destes em função do teor de umidade inicial ${ }^{2}$.

Os modelos desenvolvidos para representar o comportamento dinâmico do processo de umidificação podem ser basicamente de dois tipos: empíricos e fenomenológicos.

Os modelos empíricos geralmente são obtidos a partir de simples correlações matemáticas dos dados experimentais. SINGH e KULSHRESTHA ${ }^{9}$, PELEG $^{8}$, SOPADE e OBEKPA ${ }^{10}$ e PAN e TANGRATANAVALEE ${ }^{7}$ são exemplos de pesquisadores que utilizaram modelos empíricos.

Por sua vez, os modelos fenomenológicos consideram as etapas elementares de transferência de massa por difusão e/ou convecção; podem ser de parâmetros concentrados ou distribuídos e, geralmente, representam as principais tendências

Recebido para publicação em $7 / 3 / 2006$

Aceito para publicação em 18/7/2007 (001686)

${ }^{1}$ Departamento de Engenharia Química,

Universidade Estadual de Maringá - UEM,

Campus Universitário, Av. Colombo, 5790, Bloco D90, CEP 87020-900,

Maringá - PR, Brasil

E-mail:lmmj@deq.uem.br

* A quem a correspondencia deve ser enviada do processo, mesmo fora das condições experimentais em que foram validados. Os modelos de parâmetros concentrados não contemplam variações espaciais das propriedades físicas no sistema, enquanto que os de parâmetros distribuídos normalmente as representam, podendo, ambos, ser utilizados para simular o comportamento do grão durante a hidratação. Entretanto, via de regra, é difícil medir os perfis de umidade no interior dos grãos, o que dificulta a validação dos modelos de parâmetros distribuídos e limita a sua utilização.

Dentre os estudos desenvolvidos com modelos fenomenológicos, podem-se destacar os trabalhos de $\mathrm{HSU}^{5-6}$ e COUTINHO et al. ${ }^{4}$. HSU ${ }^{5}$ propôs um modelo fenomenológico de parâmetros distribuídos, obtido a partir de um balanço de massa diferencial num grão de soja, admitindo forma esférica de diâmetro constante, e representado por uma equação diferencial parcial resolvida numericamente. Posteriormente, $\mathrm{HSU}^{6}$ estudou a influência da temperatura na difusão de água em grãos de soja utilizando o modelo desenvolvido anteriormente, obtendo boa concordância com dados experimentais da hidratação. Ao contrário de $\mathrm{HSU}^{6}$, COUTINHO et al. ${ }^{4}$ desenvolveram um modelo de parâmetros concentrados que leva em conta o aumento do diâmetro ao longo do processo de hidratação. Esta variação do diâmetro foi obtida experimentalmente e é independente da temperatura de hidratação.

Neste trabalho, é desenvolvido um modelo fenomenológico de parâmetros concentrados para a hidratação dos grãos de soja que contempla a variação do volume dos grãos de soja durante o processo. Este modelo foi validado a partir de dados experimentais da umidade média do grão ao longo do tempo, medidos em várias temperaturas. 


\section{Material e métodos}

\subsection{Equipamento e procedimento experimental}

O equipamento utilizado neste trabalho foi o mesmo utilizado por COUTINHO et al. ${ }^{4} \mathrm{e}$ consiste em uma caixa de isopor com 0,39 $\mathrm{m}$ de comprimento, 0,25 $\mathrm{m}$ de largura, 0,17 $\mathrm{m}$ de altura e 0,05 $\mathrm{m}$ de espessura, ilustrado na Figura 1.

Dentro da caixa, foram colocados 2 recipientes com capacidade de $2 \mathrm{~L}$ de água cada. Na parte superior de cada recipiente, foi colocada uma peneira na qual se depositavam as amostras de soja com umidade em torno de $10 \%$ (b.u.).

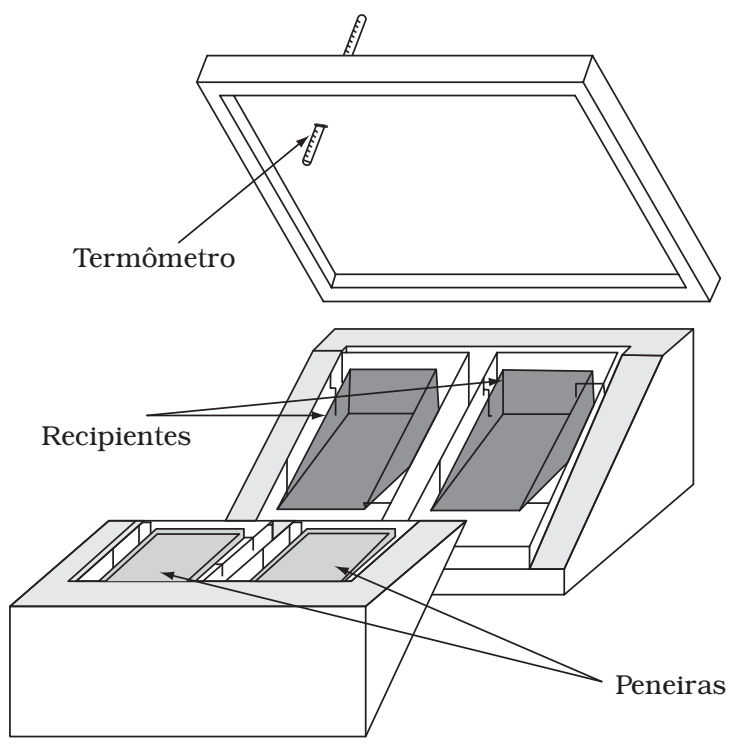

Figura 1. Sistema utilizado no experimento.

Os experimentos foram realizados conforme a seqüência detalhada abaixo:

1) Coloca-se água nos recipientes numa determinada temperatura: 15 , 30 e $49{ }^{\circ} \mathrm{C}$;

2) Adiciona-se aproximadamente $0,15 \mathrm{~kg}$ de soja em cada peneira;

3) Acompanha-se a hidratação da soja, retirando uma amostra de soja de cada recipiente em intervalos de tempo pré-determinados. Tipicamente nos instantes de tempo: 1, 5, 10, 15, 20, 25, 30, 40, 50, 60, 80, 100, 120, 150 e 180 minutos;

4) Dispõe-se cada amostra de grãos sobre papel toalha, para retirar o excesso de água superficial;

5) Divide-se cada amostra em duas parcelas: a primeira, para determinação do volume e a segunda, para determinação da umidade;

6) Avalia-se o volume e a densidade da soja por picnometria e calcula-se a variação média do volume de um grão de soja;
7) Determina-se a umidade das amostras pelo método clássico de secagem em estufa a $105^{\circ} \mathrm{C}$ durante 24 horas ou peso constante;

8) Sabendo-se que, $X=\frac{m_{\text {agua }}}{m_{\text {s.seco }}}$ a variação da massa é determinada pela Equação 1:

$$
\Delta \mathrm{m}=\mathrm{m}_{\mathrm{s} \cdot \operatorname{seco}}\left(\mathrm{X}_{\mathrm{U}}-\mathrm{X}_{\mathrm{S}}\right)
$$

\subsection{Modelagem matemática}

Partindo de um balanço de massa para a água contida na soja e admitindo-se que a concentração de água no grão seja uniforme em cada instante de tempo, obtém-se um modelo de parâmetros concentrados representado pela Equação 2.

$\frac{\mathrm{d}\left(\rho_{\text {soja }} \mathrm{V}\right)}{\mathrm{dt}}=\mathrm{K}_{\mathrm{s}} \mathrm{A}\left(\rho_{\mathrm{H}_{2} \mathrm{O}}-\rho_{\text {soja }}\right)$

sendo $\mathrm{V}$ o volume do grão, $\mathrm{K}_{\mathrm{s}} \mathrm{o}$ coeficiente de transferência de massa modificado, A a área externa do grão de soja, $\rho_{\mathrm{H}_{2} \mathrm{O}}$ a densidade da água e $\rho_{\text {soja }}$ a concentração da água na soja.

Ensaios experimentais indicam que o volume do grão de soja varia significativamente durante a hidratação, logo, variações do volume e da concentração de água na soja devem ser contempladas, conforme Equação 3.

$\rho_{\text {soja }} \frac{\mathrm{dV}}{\mathrm{dt}}+\mathrm{V} \frac{\mathrm{d} \rho_{\text {soja }}}{\mathrm{dt}}=\mathrm{K}_{\mathrm{s}} \mathrm{A}\left(\rho_{\mathrm{H}_{2} \mathrm{O}}-\rho_{\text {soja }}\right)$

A equação diferencial parcial, Equação 3, pode ser simplificada e reduzida a uma equação diferencial ordinária, Equação 7, admitindo-se que a variação do volume é diretamente proporcional à variação da massa do grão ao longo da hidratação, conforme a Equação 4.

$\Delta \mathrm{V}=\alpha \Delta \mathrm{m}$

Por sua vez, como a variação da massa $(\Delta \mathrm{m})$ se dá pela absorção de água, pode-se afirmar que $\Delta \mathrm{m}=\rho_{\text {soja }} \mathrm{V}-\rho_{\text {soja }} \mathrm{V}_{0}$, conduzindo à Equação 5.

$\mathrm{V}=\frac{\mathrm{V}_{0}\left(1-\alpha \rho_{\text {soja }_{0}}\right)}{\left(1-\alpha \rho_{\text {soja }}\right)}$

Derivando-se a Equação 5 em relação ao tempo, obtém-se a Equação 6.

$$
\frac{\mathrm{dV}}{\mathrm{dt}}=\frac{\mathrm{V}_{0}\left(1-\alpha \rho_{\text {soja }_{0}}\right) \alpha \frac{\mathrm{d} \rho_{\text {soja }}}{\mathrm{dt}}}{\left(1-\alpha \rho_{\text {soja }}\right)^{2}}
$$

Admitindo-se que os grãos de soja sejam esféricos e substituindo-se a Equação 6 na Equação 3, obtém-se a Equação 7.

$\frac{\mathrm{d} \rho_{\text {soja }}}{\mathrm{dt}}=\frac{6 \mathrm{~K}_{\mathrm{s}}}{\mathrm{C}}\left(\rho_{\mathrm{H}_{2} \mathrm{O}}-\rho_{\text {soja }}\right)\left(1-\alpha \rho_{\text {soja }}\right)^{4 / 3}$ 
em que:

$\mathrm{C}=\left(\frac{6}{\pi} \mathrm{V}_{0}\left(1-\alpha \rho_{\text {soja }_{0}}\right)\right)^{1 / 3}$

Assumindo-se uma variação exponencial do coeficiente de transferência de massa $\left(\mathrm{K}_{\mathrm{s}}\right)$ em função da concentração de água na soja de acordo com a Equação 9 e substituindo-a na Equação 7, obtém-se a Equação 10 que representa o modelo na sua forma final.

$\mathrm{K}_{\mathrm{s}}=\mathrm{C}_{1} \cdot \mathrm{e}^{\left(\mathrm{C}_{2} \cdot \mathrm{P}_{\mathrm{soja}}\right)}$

$\frac{\mathrm{d} \rho_{\text {soja }}}{\mathrm{dt}}=\frac{6\left(\mathrm{C}_{1} \cdot \mathrm{e}^{\left(\mathrm{C}_{2} \cdot \rho_{\text {soja }}\right)}\right)}{\mathrm{C}}\left(\rho_{\mathrm{H}_{2} \mathrm{O}}-\rho_{\text {soja }}\right)\left(1-\alpha \rho_{\text {soja }}\right)^{4 / 3}$

\subsection{Ajuste individual do modelo matemático}

Os parâmetros ajustáveis do modelo $\left(\mathrm{C}_{1} \mathrm{e} \mathrm{C}_{2}\right)$ foram obtidos por meio de integração numérica da Equação 10, com a condição inicial: $t=0, \rho_{\text {soja }}=\rho_{0}$, no programa MATLAB (versão 5.1), utilizando-se a rotina fmins.m para minimizar o resíduo quadrático: $\phi^{2}=\sum\left(\rho_{\text {calc }}-\rho_{\text {exp }}\right)^{2}$

\section{Resultados e discussão}

\subsection{Variação do volume ao longo da hidratação}

A Figura 2 revela que o comportamento da variação do volume $\left(\mathrm{V}-\mathrm{V}_{0}\right)$ em relação à variação da massa do grão $\left(\mathrm{m}-\mathrm{m}_{0}\right)$ ao longo da hidratação é linear, independente da temperatura, e pode ser satisfatoriamente ajustado por uma reta, cujo coeficiente angular $(\alpha)$ é igual a $1,04 \times 10^{-3} \mathrm{~m}^{3} \cdot \mathrm{kg}^{-1}$, que por sua vez é muito próximo ao volume específico da água (1,0 x $\left.10^{-3} \mathrm{~m}^{3} \cdot \mathrm{kg}^{-1}\right)$, conforme esperado.

Comprovado que o valor de $\alpha$ corresponde aproximadamente ao volume específico da água, procedeu-se à simplificação do modelo (Equação 10), considerando-se que $\alpha=1$, transformando-o em um modelo com apenas dois parâmetros $\left(\mathrm{C}_{1}\right.$ e $\left.\mathrm{C}_{2}\right)$.

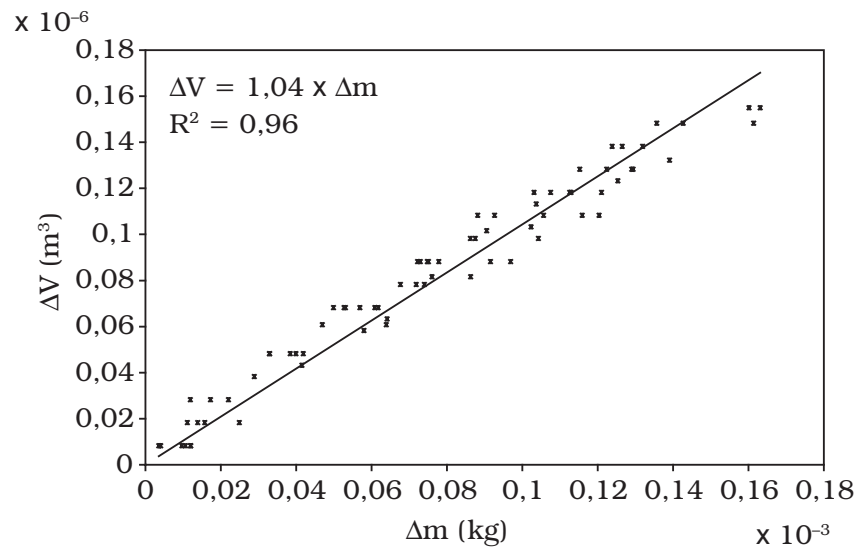

Figura 2. Variação do volume em função da variação da massa do grão.

\subsection{Ajuste do modelo matemático}

Na Figura 3 são apresentados os resultados das simulações do modelo matemático juntamente com valores experimentais para as temperaturas de 15,30 e $49^{\circ} \mathrm{C}$. A análise destes resultados confirma que o modelo pôde representar adequadamente as principais tendências do processo de hidratação, com desvios da ordem de $\pm 5 \%$, conforme se pode observar na Figura 4 .

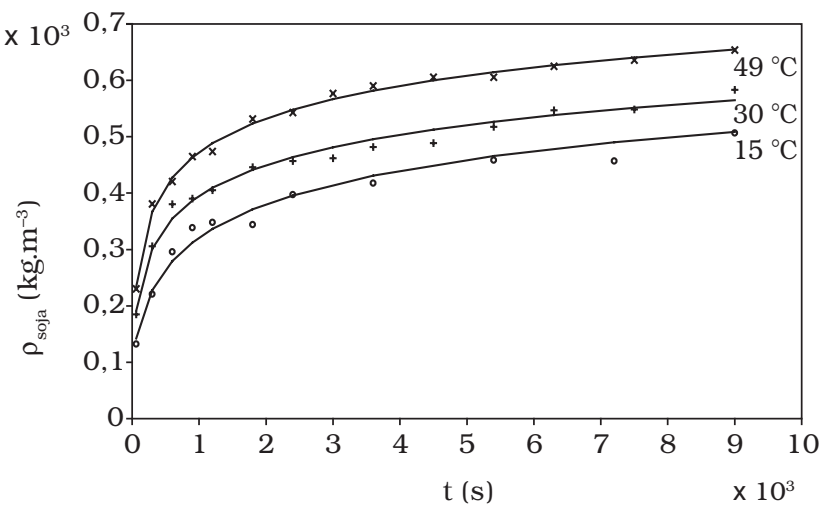

Figura 3. Previsões do modelo frente a dados experimentais.

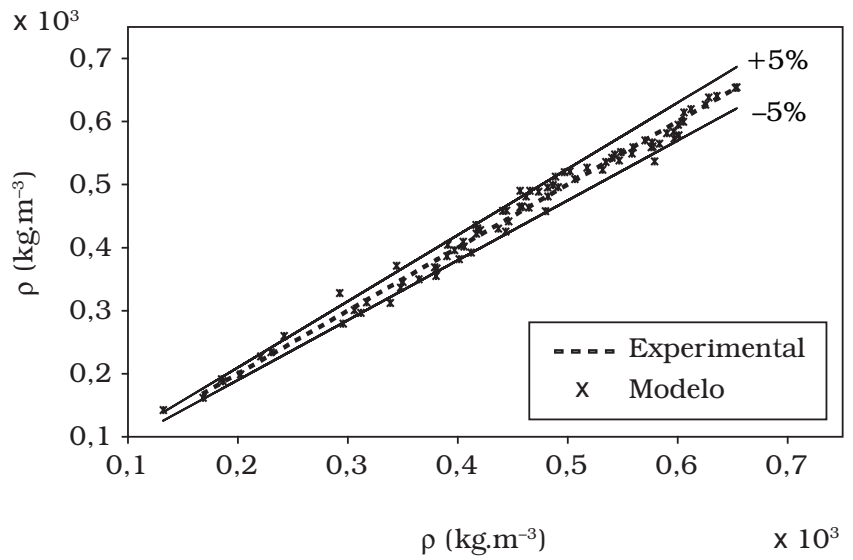

Figura 4. Desvios do modelo.

Na Tabela 1 são apresentados os valores dos parâmetros utilizados nas simulações que originaram os dados contidos nas Figuras 3 e 4. Estes parâmetros foram ajustados pelo método dos mínimos quadrados, segundo metodologia descrita previamente.

Tabela 1. Parâmetros do modelo.

\begin{tabular}{ccc}
\hline $\mathrm{T}\left({ }^{\circ} \mathrm{C}\right)$ & $\mathrm{C}_{1}$ & $\mathrm{C}_{2}$ \\
\hline 15 & $2,323 \times 10^{-6}$ & $-7,651 \times 10^{-3}$ \\
30 & $8,014 \times 10^{-6}$ & $-8,814 \times 10^{-3}$ \\
49 & $1,229 \times 10^{-5}$ & $-7,386 \times 10^{-3}$ \\
\hline
\end{tabular}




\subsection{Comportamento do coeficiente de transferência de massa aparente ao longo da hidrataçáo}

É importante salientar que usualmente o coeficiente de transferência de massa é definido a partir da Equação 11.

$\mathrm{N}_{\mathrm{A}}=\mathrm{K}\left(\rho_{\text {soja }}^{\mathrm{eq}}-\rho_{\text {soja }}\right)$

Entretanto, neste trabalho e em COUTINHO et al. ${ }^{4}$, aplicou-se o conceito de um coeficiente de transferência de massa aparente, $\mathrm{K}_{\mathrm{s}}$, definido por uma equação similar (Equação 12), no desenvolvimento do modelo matemático, Equação 2.

$\mathrm{N}_{\mathrm{A}}=\mathrm{K}_{\mathrm{s}}\left(\rho_{\mathrm{H}_{2} \mathrm{O}}-\rho_{\text {soja }}\right)$

A utilização de $\rho_{\mathrm{H}_{2} \mathrm{O}}$ em vez de $\rho_{\text {soja }}^{\text {eq }}$ foi concebida a fim de reduzir o número de parâmetros ajustáveis do modelo. Esta simplificação conduz a um modelo, cujo coeficiente de transferência de massa aparente $\left(\mathrm{K}_{\mathrm{s}}\right)$ difere daquele usado tradicionalmente (K), mas não compromete a qualidade da representação do modelo conforme se pode observar na Figura 3.

O coeficiente de transferência de massa aparente, $K_{s}$, foi correlacionado em função da concentração de água na soja, conforme Figura 5. Observa-se que o valor de $\mathrm{K}_{\mathrm{s}}$ diminui significativamente com o aumento da concentração. O coeficiente de transferência de massa aparente tende a zero quando $\rho_{\text {soja }}$ tende ao valor de equilíbrio. Este comportamento era esperado, pois o valor de $\rho_{\text {soja }}$ de equilíbrio será sempre menor do que a densidade da água, portanto, para que o fluxo mássico (Equação 2) seja nulo (o que ocorre no equilíbrio), $\mathrm{K}_{\mathrm{s}}$ deverá ser igual a zero. Nota-se também que o $\mathrm{K}_{\mathrm{s}}$ aumenta com o aumento da temperatura.

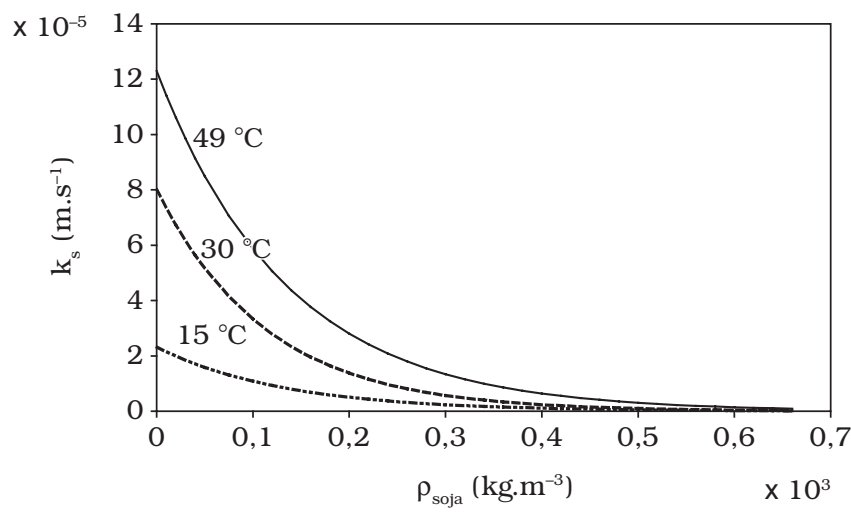

Figura 5. Comportamento de $\mathrm{K}_{\mathrm{s}}$ com a concentração de água.

\section{Conclusões}

O modelo pôde representar bem o processo de hidratação, com um erro máximo em torno de $5 \%$.

A hipótese simplificadora adotada no modelo de que a variação do volume do grão é proporcional à variação da massa do grão de soja é coerente e o valor de foi aproximadamente igual ao volume específico da água $\left(1,0 \times 10^{-3} \mathrm{~m}^{3} \cdot \mathrm{kg}^{-1}\right)$.

A variação do coeficiente de transferência de massa aparente $\left(\mathrm{K}_{\mathrm{s}}\right)$ ao longo da hidratação pôde ser representada satisfatoriamente por uma função exponencial: $\mathrm{K}_{\mathrm{s}}=\mathrm{C}_{1} \cdot \mathrm{e}^{\left(\mathrm{C}_{2} \cdot \rho_{\text {soja }}\right)}$
O coeficiente de transferência de massa aparente $\left(\mathrm{K}_{2}\right)$ aumenta conforme se eleva a temperatura e, independentemente desta, tende a zero quando a concentração de água na soja se aproxima do valor de equilíbrio.

\section{Nomenclatura}

$\begin{array}{lll}\mathrm{A} & \text { Área } & \left(\mathrm{m}^{2}\right) \\ \mathrm{C}_{1} & \text { parâmetro de ajuste do modelo Fenomenológico } & \left(\mathrm{m}^{-\mathrm{s}^{-1}}\right) \\ \mathrm{C}_{2} & \text { parâmetro de ajuste do modelo Fenomenológico } & \left(\mathrm{m}^{3} \cdot \mathrm{kg}^{-1}\right) \\ \mathrm{K}_{\mathrm{s}} & \text { coeficiente de transferência de massa modificado } & \left(\mathrm{m} . \mathrm{s}^{-1}\right) \\ \mathrm{m} & \text { massa do grão } & (\mathrm{kg}) \\ \mathrm{m}_{\text {água }} & \text { massa de água no grão } & (\mathrm{kg}) \\ \mathrm{m}_{\mathrm{s} . \mathrm{seco}} & \text { massa de sólido seco no grão } & (\mathrm{kg}) \\ \mathrm{N}_{\mathrm{a}} & \text { fluxo mássico } & \\ \mathrm{t} & \text { Tempo } & (\mathrm{s}) \\ \mathrm{T} & \text { Temperatura } & \left({ }^{\circ} \mathrm{C}\right) \\ \mathrm{V} & \text { volume do grão } & \left.\text { (m }{ }^{3}\right) \\ \mathrm{X}_{\mathrm{e}} & \text { umidade de equilíbrio em base seca } & \text { (adim.) } \\ \mathrm{X} & \text { umidade em base seca } & \text { (adim.) } \\ \mathrm{X}_{0} & \text { umidade inicial em base seca } & \text { (adim.) } \\ \mathrm{X}_{\mathrm{U}} & \text { umidade em base seca do grão após a umidifi- } & \text { (adim.) } \\ & \text { cação } & \\ \mathrm{X}_{\mathrm{s}} & \text { umidade em base seca do grão antes da umidi- } & \text { (adim.) } \\ & \text { ficação } & \end{array}$

\subsection{Símbolos gregos}

$\alpha \quad$ coeficiente angular da Equação $4 \quad$ (adim.)

$\rho_{\text {calc }}$ concentração de água na soja calculada pelo modelo (kg.m ${ }^{-3}$ )

$\rho_{\text {esp }}$ concentração de água na soja obtida experimentalmente (kg.m $\left.{ }^{-3}\right)$

$\rho_{\mathrm{H}_{2} \mathrm{O}}$ densidade da água $\quad\left(\mathrm{kg} \cdot \mathrm{m}^{-3}\right)$

$\rho_{\mathrm{soja}}$ concentração da água na soja $\quad\left(\mathrm{kg} \cdot \mathrm{m}^{-3}\right)$

$\rho_{\mathrm{e}} \quad$ concentração de água na soja no equilíbrio $\quad\left(\mathrm{kg} \cdot \mathrm{m}^{-3}\right)$

$\rho_{0} \quad$ concentração inicial de água na soja $\quad\left(\mathrm{kg} \cdot \mathrm{m}^{-3}\right)$

$\phi^{2}$ desvio quadrático (adim.)

\section{Referências bibliográficas}

1. BARROZO, M. A. S.; CALADO, V. M. A.; CALÇADA, L. A. Secagem de Soja em Leito Deslizante e Recirculação Pneumática: Influência da Umidificação Artificial, In: XIX Encontro Sobre Escoamento em Meios Porosos. Anais... Campinas, SP, v. I, p. 327-337, 1991.

2. BECKERT, O. P.; MIGUEL, M. H.; MARCOS FILHO, J. Absorção de Água e Potencial Fisiológico em Sementes de Soja de Diferentes Tamanhos. Scientia Agrícola, v. 57, n. 4, p. 671-675, 2000.

3. CALADO, V. M. A. (1993) Modelagem e Simulação de Secadores em Leitos Fixo e Deslizante. Tese D.Sc., COPPE, UFRJ.

4. COUTINHO, M. R.; OMOTO, E. S. ANDRADE, C. M. G. e JORGE, L M. M. Modelagem e Validação da Hidratação de Grãos de Soja. Ciênc. Tecnol. Aliment., v. 25, n. 3, p. 603-610, Campinas, 2005.

5. HSU, K. H. A Diffusion Model with a Concentration-Dependent Diffusion Coefficient for Describing Water Movement in Legumes During Soaking. J. of Food Sci., v. 48, n. 2, p. 618-622 e 645, 1983.

6. HSU, K. H. Effect of Temperature on Water Diffusion in Soybean. J. of Food Sci., v. 48, n. 4, p. 1364 e 1365, 1983. 
7. PAN, Z.; TANGRATANAVALEE, W. Characteristics of soybean as affected by soaking conditions, Lebensm. -Wiss. U.- Technol., v. 36, n. 1, p. 143-151, 2003.

8. PELEG, M. An Empirical Model for the Description of Moisture Sorption Curves. J. of Food Sci., v. 53, n. 4, p. 1216-1217 e 1219, 1988.

9. SINGH, B. P. N.; KULSHRESTHA, S. P. Kinetcs of Water Sorption by Soybean and Pigeonpea Grains. J. of Food Sci., v. 52, n. 6, p. 1538-1541 e 1544, 1987.
10. SOPADE, P. A.; OBEKPA, J. A. Modelling Water Absorption in Soybean, Cowpea and Peanuts at Three Temperatures Using Peleg's Equation. J. of Food Sci., v. 55, n. 4, p. 1085-1087, 1990.

11. WANG, H. L. et al. Hydration of Whole Soybeans Affects Solids Losses and Cookint Quality. J. of Food Sci., v. 44, n. 5, p. 1510-1513, 1979. 